\title{
Nanojoining and tailoring of current-voltage characteristics of metal-P type semiconductor nanowire heterojunction by femtosecond laser irradiation
}

Cite as: J. Appl. Phys. 127, 184901 (2020); doi: 10.1063/5.0004272

Submitted: 10 February 2020 . Accepted: 29 April 2020 .

Published Online: 11 May 2020

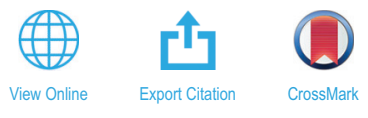

M. Xiao, ${ }^{1,2, a)}$ (D) L. Lin, ${ }^{3}$ S. Xing, ${ }^{4}$ (D) J. Feng, ${ }^{2,5}$ Z. Hui, ${ }^{2,6}$ S. Wang, ${ }^{2,7}$ (D) W. W. Duley, ${ }^{2,8}$ and Y. N. Zhou ${ }^{1,2}$

\begin{abstract}
AFFILIATIONS
${ }^{1}$ Department of Mechanical and Mechatronics Engineering, University of Waterloo, Waterloo, Ontario N2L 3G1, Canada

${ }^{2}$ Centre for Advanced Materials Joining, University of Waterloo, Waterloo, Ontario N2L 3G1, Canada

${ }^{3}$ Empa, Swiss Federal Laboratories for Materials Science and Technology, Überlandstrasse 129, 8600 Dübendorf, Switzerland

${ }^{4}$ Department of Mechanical Engineering, State Key Laboratory of Tribology, Tsinghua University, Beijing 100084,

People's Republic of China

${ }^{5}$ State Key Laboratory of Advanced Welding and Joining, Harbin Institute of Technology, Harbin 150001,

People's Republic of China

${ }^{6}$ School of Materials Science and Technology, China University of Geosciences, Beijing 100083, People's Republic of China

${ }^{7}$ Laser Micro/Nano Fabrication Laboratory, School of Mechanical Engineering, Beijing Institute of Technology, Beijing 100081,

People's Republic of China

${ }^{8}$ Department of Physics and Astronomy, University of Waterloo, Waterloo, Ontario N2L 3G1, Canada
\end{abstract}

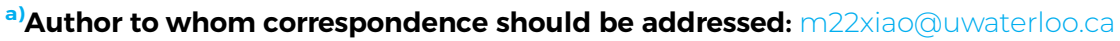

\begin{abstract}
Selective engineering of the interface between nanoscale components and the electrical properties of heterojunctions is key to the development of next-generation nanoscale circuit elements. In this paper, we show how laser processing of a metal-P type semiconductor nanoscale heterojunction between $\mathrm{Ag}$ and $\mathrm{CuO}$ nanowires can be used to control the nature of the electrical contact by reducing the Schottky barrier at the $\mathrm{Ag}-\mathrm{CuO}$ interface to Ohmic contact. Elimination of the Schottky barriers occurs in response to lattice matching of $\mathrm{Ag}(111) \| \mathrm{CuO}(111)$ planes at the interface induced by controlled irradiation with femtosecond (fs) laser pulses. An interdiffusion region with a mixed $\mathrm{Ag} / \mathrm{CuO}$ composition is also present over a localized area of the interface between the $\mathrm{Ag}$ and $\mathrm{CuO}$ nanowires after fs laser processing, but both $\mathrm{Ag}$ and $\mathrm{CuO}$ nanowires remain crystalline away from the heterojunction. In addition, the $\mathrm{Ag}$ nanowire becomes totally embedded in the larger $\mathrm{CuO}$ nanowire after irradiation. Fabricated nanowire devices from $\mathrm{Ag}-\mathrm{CuO}$ nanowire heterojunctions transition from a double-Schottky contact configuration prior to laser processing to a rectifying behavior as irradiation time increases. This study illustrates that fs laser processing can be highly effective in the engineering of electrical performance in metal-semiconductor nanoscale heterojunction devices.
\end{abstract}

Published under license by AIP Publishing. https://doi.org/10.1063/5.0004272

\section{INTRODUCTION}

The metal-semiconductor interface is fundamental in semiconductor physics and has been widely studied since the original development of semiconductor devices over 100 years ago. Moreover, next-generation electronic devices based on nanoscale materials incorporated in one-dimensional semiconductor structures are now attracting much attention as replacements for existing compositions involving Si-technology. However, the high surface-to-volume ratio inherent in nanomaterials implies that the operating characteristics of these devices are strongly dependent on the nature of interfaces 
between the nanomaterial and electrodes as well as with other nanoscale materials in the device. This invariably leads to the variations in device performance, even for those with the same metalsemiconductor combination, ${ }^{1-6}$ limiting the future development and application of nanoscale electronic devices. These limitations are also commonly encountered in the development of electronic devices based on 2D materials where standardizing the contact/interface between semiconductor building blocks and electrodes/other components is fundamental to obtaining reproducible performance. ${ }^{7-11}$ New technologies for the in situ engineering of nanoscale interfaces to achieve optimized performance are then a high priority.

One method, involving plasmonic engineering by laser irradiation, has recently shown great potential. ${ }^{12-15}$ Irradiation with ultrafast laser pulses has been widely used for the joining of metallic nanomaterials such as $\mathrm{Ag}, \mathrm{Cu}$, and $\mathrm{Au}$ nanorods or nanowires for the production of transparent electrodes and conductive heaters, as well as other devices. ${ }^{16-18}$ Some advantages associated with the use of plasmonic effects during laser irradiation of these metallic nanomaterials include high spatial resolution, high energy efficiency, and increased scalability for mass production. ${ }^{19,20}$ However, a detailed evaluation of the use of the plasmonic effect in tailored engineering of the metal-semiconductor nanoscale interface is still limited. Optimization of the properties of the metal-semiconductor interface is fundamental to the reliable operation of most functional devices including the transistor, memory circuits, and other more complex circuits as the performance of nanoscale devices relies to a large extent on the control over interfacial structures and the type and distribution of interfacial states in metal-semiconductor structures. Laser induced plasmonic effects offer the advantage of intense highly localized energy input at the metal-semiconductor interface without attendant heating of the surrounding nanomaterials. Selective plasmonic heating in functional units can produce nano-scale structural changes in individual interfacial layers enabling nano-engineering of electrical characteristics and performance.

In this paper, we report a study of the evolution of the structure and composition of an $\mathrm{Ag}-\mathrm{CuO}$ nanowire heterojunction under low fluence femtosecond (fs) laser irradiation, and the role of this irradiation plays in determining the electrical performance of the resulting metal-P type semiconductor nanoscale heterojunction. In a surprising result, we find that the Ag nanowire becomes embedded in the $\mathrm{CuO}$ nanowire in response to plasmonic heating induced by fs laser irradiation. Characterization of this structure using high-resolution transmission electron microscopy (HRTEM) reveals an interdiffusion region, which maintains individual crystalline microstructures at the $\mathrm{Ag}-\mathrm{CuO}$ hetero-interface. Due to this inter-diffusion region, a transition from the original double-Schottky behavior for the fabricated $\mathrm{Ag}-\mathrm{CuO}$ nanowire device to a single Schottky barrier can be induced by fs laser irradiation. This suggests that fs laser irradiation is a useful new technology for nanoscale engineering of metal-semiconductor interfaces for the optimization of the electrical performance of nanoscale devices.

\section{EXPERIMENTAL}

$\mathrm{CuO}$ nanowires were obtained by heating a clean $\mathrm{Cu}$ foil at $500{ }^{\circ} \mathrm{C}$ for $4 \mathrm{~h}$ in air, ${ }^{21}$ while $\mathrm{Ag}$ nanowires were synthesized by standard chemical reduction methods. ${ }^{22}$ Both $\mathrm{CuO}$ and Ag nanowires were diluted in ethanol before device fabrication. The $\mathrm{CuO}$ and $\mathrm{Ag}$ nanowires were then deposited on a silicon wafer using a sequential drop-cast process. After drying in air, samples were irradiated with low fluence femtosecond (fs) laser pulses ( $35 \mathrm{fs}$ pulse duration, $1000 \mathrm{~Hz}$ frequency, $800 \mathrm{~nm}$ wavelength) for different lengths of time. Figure 1 shows a schematic diagram of the laser irradiation experimental setup. The diameter of the beam from the fs laser was $30 \mathrm{~mm}$. The laser beam was then focused on a $2 \mathrm{~mm}$ diameter spot size using a convex lens with a $20 \mathrm{~cm}$ focal length. Scanning electron microscopy (SEM) was used for characterization of the $\mathrm{Ag}-\mathrm{CuO}$ nanowire heterojunction, while a focused ion beam (FIB, FEI Helios 660) system was utilized for the preparation of cross-sectional lamellae for imaging with transmission electron microscopy (TEM, JEOL2200FS). A focused ion beam system (FIB, Zeiss NVision 40) was used to deposit W electrodes to connect the $\mathrm{Ag}-\mathrm{CuO}$ nanowire heterojunction for electrical characterization in a two-terminal configuration using a Keithley 4200A semiconductor characterization system (SCS). The electric field distribution for the $\mathrm{Ag}-\mathrm{CuO}$ nanowire heterojunction under laser irradiation was further simulated by the commercial finite element method (FEM) software with an RF module (COMSOL Multiphysics, 5.3a).

\section{RESULTS AND DISCUSSION}

Figure 2 shows the SEM images of the Ag-CuO nanowire heterojunction after fs irradiation at the fluence of $10 \mathrm{~mJ} / \mathrm{cm}^{2}$. Since both the $\mathrm{Ag}$ and $\mathrm{CuO}$ nanowires were not selectively oriented during deposition on the silicon wafer, while the laser beam is linearly polarized, the direction of the laser electric field is randomized relative to any of the nanowire components. Previous studies have shown that the direction of laser polarization greatly affects energy deposition in a nanowire structure and has a significant effect on the quality of joining. In particular, an earlier study of the joining of $\mathrm{Ag}$ and $\mathrm{TiO}_{2}$ nanowires by fs laser irradiation demonstrates the relevance of the angle between the long axis of the Ag nanowire and the direction of laser polarization. It was found that the enhancement in the plasmonic electric field in an Ag nanowire is the largest when the direction of laser polarization is parallel to the long axis of the Ag nanowire. ${ }^{23}$ This effect can be seen in SEM images in the present study as $\mathrm{Ag}-\mathrm{CuO}$ nanowire heterojunctions are facilitated in structures with orientations such that the long axis of the Ag nanowire is parallel to the laser polarization. Under these conditions, the plasmonic heating efficiency is maximized, and fs laser radiation is strongly coupled into the Ag nanowire via the surface plasmon resonance. The Ag nanowires were found to separate into a linear array of co-joined/separated nanospheres when irradiation occurred at a higher fluence. ${ }^{24}$ The $10 \mathrm{~mJ} / \mathrm{cm}^{2}$ fluence used in these experiments was chosen to reveal the evolution of joining of $\mathrm{Ag}-\mathrm{CuO}$ nanowire heterojunctions under a variety of geometrical conditions and to relate subsequent electrical performance to irradiation times. As shown in Fig. 2(a), it is apparent that the Ag nanowire is melted following irradiation with the junction structure embedded into the $\mathrm{CuO}$ nanowire, suggesting that the $\mathrm{Ag}$ and $\mathrm{CuO}$ nanowires are locally fused at the junction. The material in either of the nanowires does not show any structural change outside the junction region. The entire Ag nanowire is 


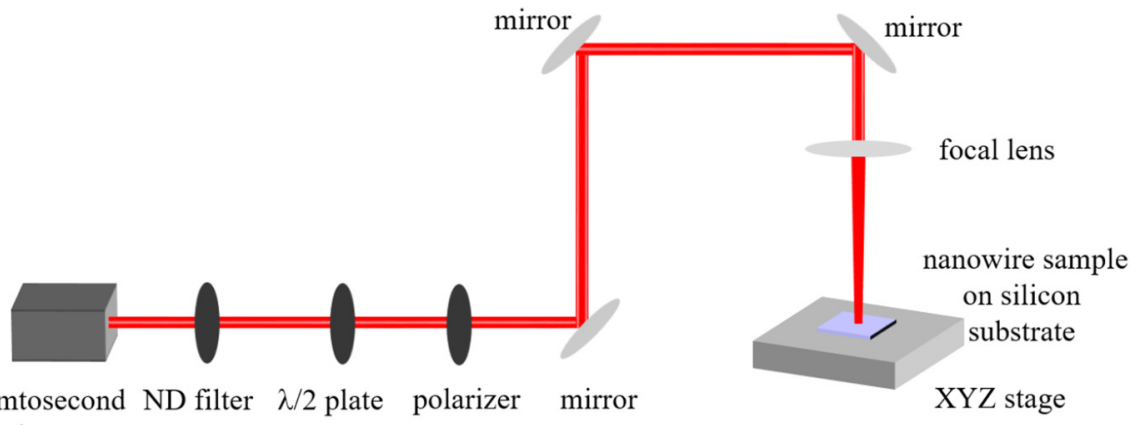

FIG. 1. Schematic diagram for the fs laser irradiation experiment setup. embedded in the larger $\mathrm{CuO}$ nanowire in the region of the overlap under short duration fs laser irradiation [Figs. 2(a) and 2(b)]. It should be noted that the Ag nanowires are also joined under the same irradiation conditions. An increase in the irradiation time enhances the embedding effect but is also accompanied by breakage of the Ag nanowire outside the region of the overlap [Fig. 2(c)]. Under these conditions, an $\mathrm{Ag}-\mathrm{CuO}-\mathrm{Ag}$ nanowire heterojunction can also be formed as shown in the inset of Fig. 2(c). Additional irradiation causes the Ag nanowire to melt into a series of small nanospheres due to the Raleigh instability from localized heating, ${ }^{25,26}$ eventually breaking the $\mathrm{Ag}-\mathrm{CuO}$ nanowire heterojunction [Fig. 2(d)].

To study the joining mechanism of the $\mathrm{Ag}-\mathrm{CuO}$ nanowire heterojunction under fs laser irradiation, a heterojunction subjected to laser irradiation for $20 \mathrm{~s}$ was cut using FIB along the direction of the long axis of the $\mathrm{CuO}$ nanowire. The TEM lamella was prepared by a FIB thinning process attached on a Mo based grid. Figure 3(a) clearly shows that the Ag nanowire is almost totally embedded into the $\mathrm{CuO}$ nanowire structure, in agreement with the SEM characterization results. Figure 3(b) shows the SEM images of the $\mathrm{Ag}-\mathrm{CuO}$
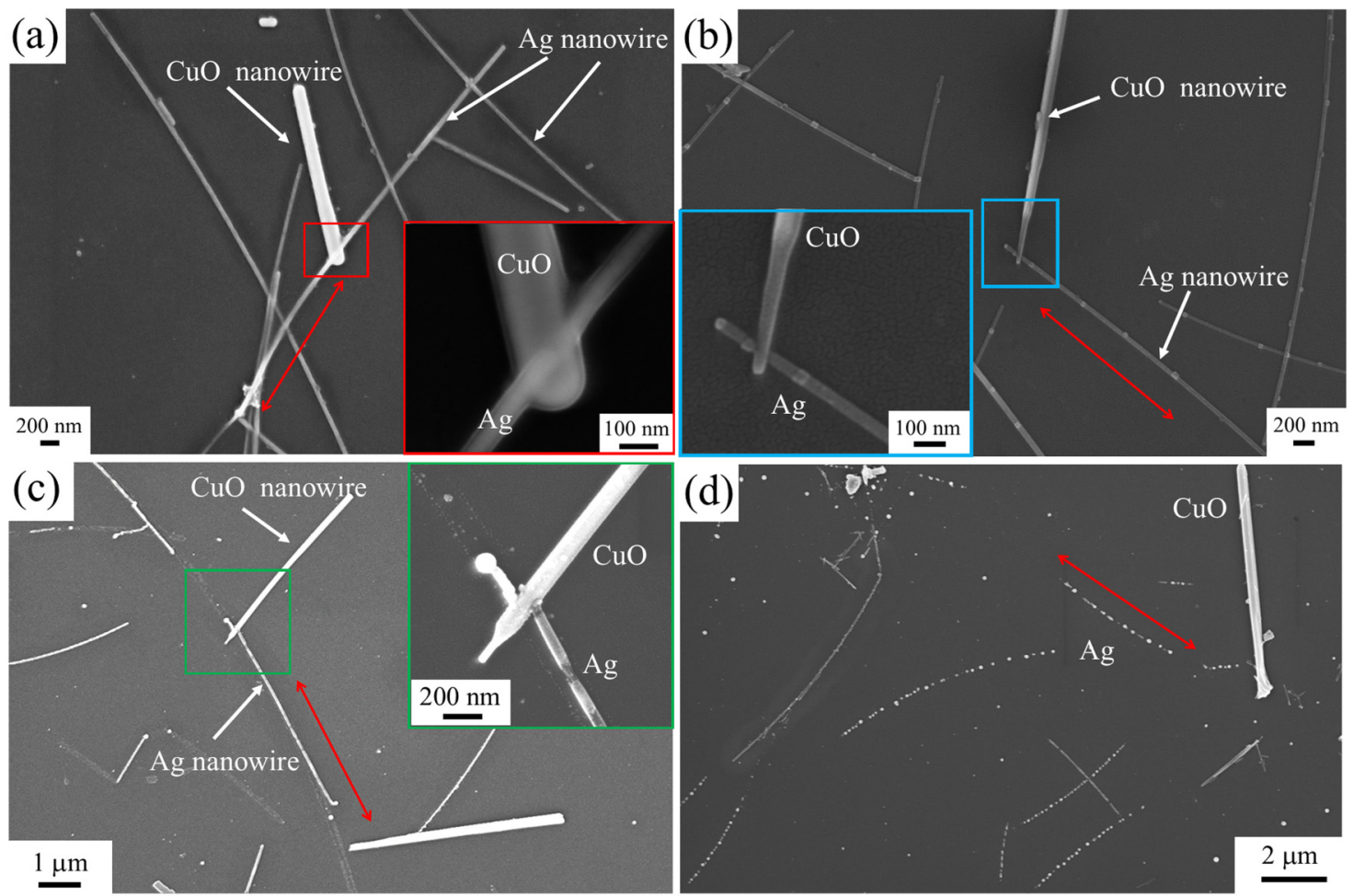

FIG. 2. Characterization of $\mathrm{Ag}-\mathrm{CuO}$ nanowire heterojunction following fs laser irradiation for different times. The fluence was $10 \mathrm{~mJ} / \mathrm{cm}^{2}$. The irradiation time duration was (a) $10 \mathrm{~s}$, (b) $20 \mathrm{~s}$, (c) $30 \mathrm{~s}$, and (d) $50 \mathrm{~s}$. Double red arrows indicate the polarization direction for the laser irradiation experiment. 


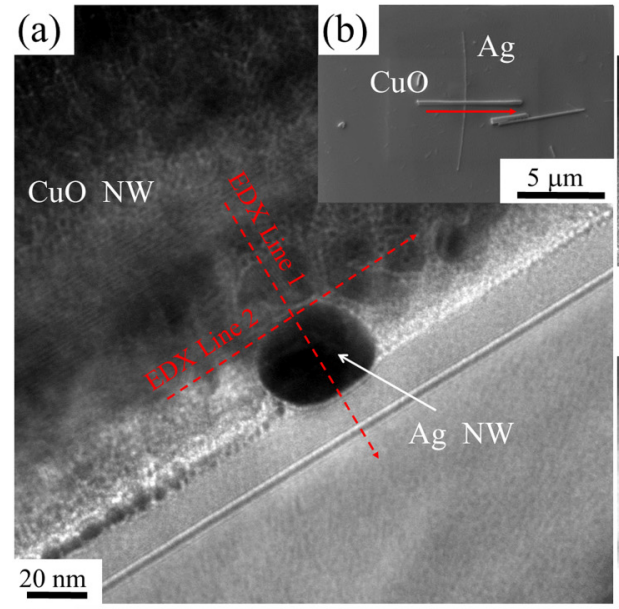

(i)

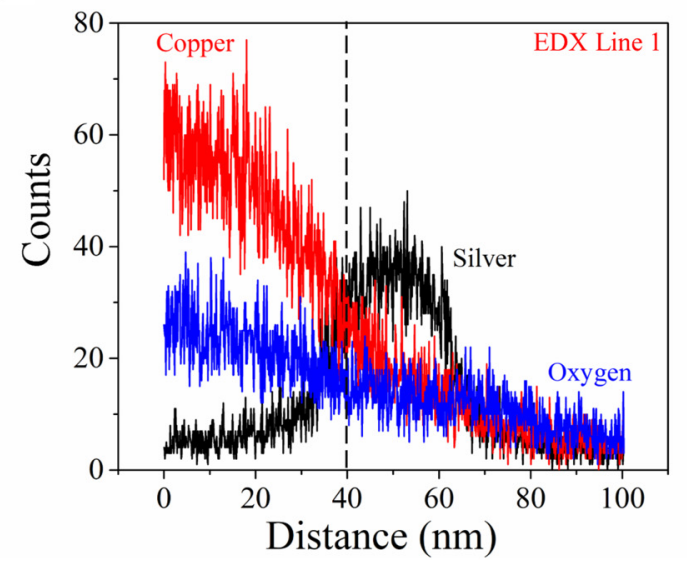

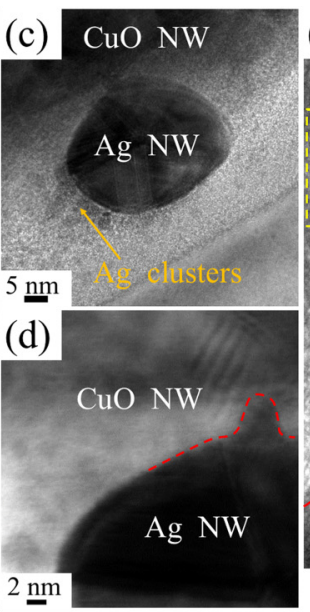
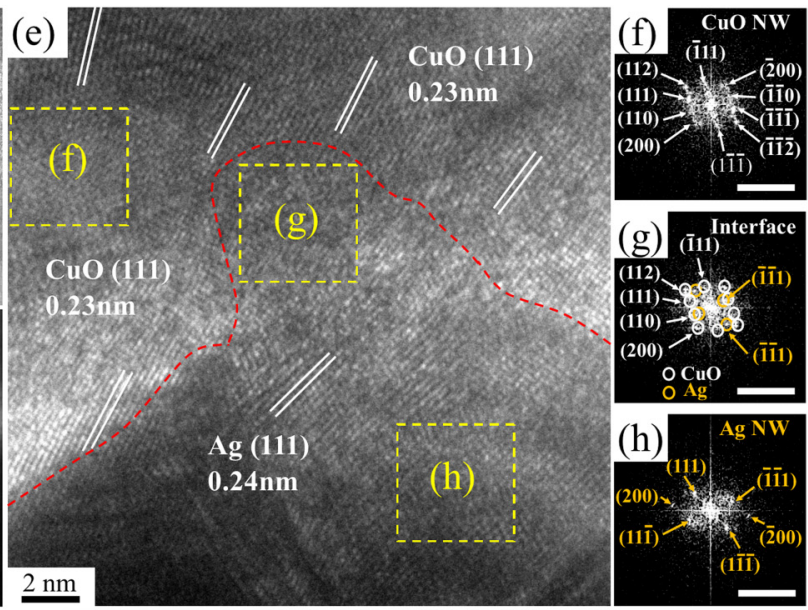

(j)

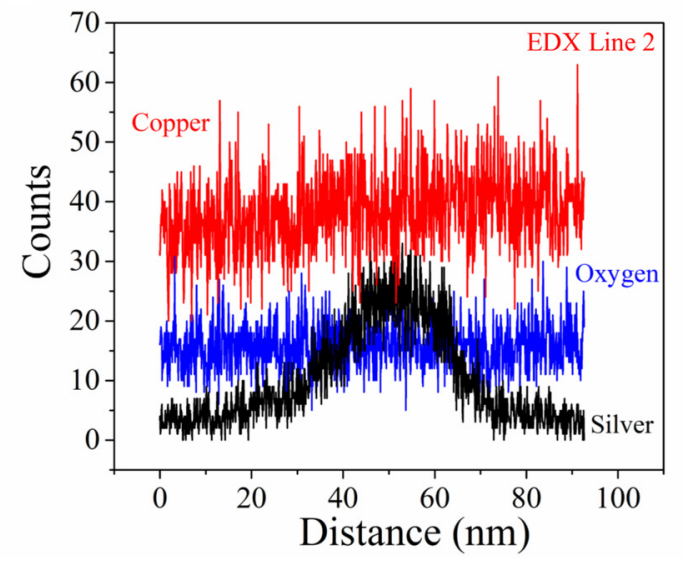

FIG. 3. TEM characterization of the Ag-CuO nanowire heterojunction after fs laser irradiation. (a) TEM image of a cross section through the Ag-CuO nanowire heterojunction, (b) SEM image of the Ag-CuO nanowire heterojunction after fs laser irradiation for $20 \mathrm{~s}$ at a fluence of $10 \mathrm{~mJ} / \mathrm{cm}^{2}$ showing the direction of the FIB cut (red arrow), (c) magnified TEM image of the $\mathrm{Ag}-\mathrm{CuO}$ nanowire heterojunction showing the presence of $\mathrm{Ag}$ clusters, and (d) TEM image showing the Ag protrusion into the CuO nanowire in the region of the $\mathrm{Ag}-\mathrm{CuO}$ nanowire heterojunction. (e) HRTEM imaging at the interface of the Ag-CuO nanowire heterojunction. (f)-(h) FFT of various selected regions in (e) including (f) the $\mathrm{CuO}$ nanowire region, (g) $\mathrm{CuO}$ and $\mathrm{Ag}$ inter-diffusion regions, and (h) the Ag nanowire region. All the scale bars are $101 / \mathrm{nm}$ for (f)-(h). (i) EDX spectrum for copper, silver, and oxygen elemental distributions along Line 1 as indicated in (a). The dashed line indicates the boundary between CuO and Ag. (j) EDX spectrum for copper, silver, and oxygen elemental distributions along Line 2 as indicated in (a).

nanowire heterojunction for the FIB cutting process, and the red arrow indicates the direction for the cutting process along the long axis of the $\mathrm{CuO}$ nanowire. Furthermore, TEM imaging reveals a few isolated clusters on the outside of the Ag nanowire [Fig. 3(c)]. This composition is confirmed as Ag by indexing the diffraction pattern from the high-resolution TEM image. Furthermore, a part of the $\mathrm{Ag}$ nanowire is also observed to protrude into the $\mathrm{CuO}$ nanowire [Fig. 3(d)], indicating that the $\mathrm{Ag}-\mathrm{CuO}$ interface becomes unstable at some point in the fs laser irradiation process. HRTEM imaging [Fig. 3(e)] shows a magnified view of the interface between $\mathrm{Ag}$ and $\mathrm{CuO}$ as well as the $\mathrm{Ag}$ protrusion. The protrusion is also indicative of the diffusion of $\mathrm{Ag}$ into the $\mathrm{CuO}$ region. Furthermore, it is evident that $\mathrm{CuO}$ and $\mathrm{Ag}$ remain crystalline away from the interface, suggesting that low fluence fs laser irradiation does not modify the overall composition or structure of the nanowires. At the interfacial region, the $\mathrm{CuO}$ is indexed as the (111) plane with a plane spacing of $0.23 \mathrm{~nm}$, which is almost identical to the $0.24 \mathrm{~nm}$ spacing in the (111) plane of the Ag nanowire. This coincidence ensures that lattice matching in the $\mathrm{Ag}-\mathrm{CuO}$ nanowire heterojunction naturally occurs at the $\operatorname{Ag}(111) \| \mathrm{CuO}(111)$ interface. Fast Fourier Transform (FFT) processing of the $\mathrm{CuO}$, Ag protrusion, and Ag regions are given in Figs. 3(f)-3(h) and confirms that the single crystalline structure of $\mathrm{CuO}$ and $\mathrm{Ag}$ in each nanowire away from the heterojunction remains unchanged after laser irradiation. The diffraction pattern of $\mathrm{Ag}$, as well as that of $\mathrm{CuO}$, is seen within the $\mathrm{Ag}$ protrusion, implying that $\mathrm{Ag}$ and $\mathrm{CuO}$ elements inter-diffuse in the protrusion region while maintaining their own microstructures. This is further evidenced by the scanning Energy 
Dispersive X-Ray (EDX) results along the two lines as indicated in Fig. 3(a) under the Scanning Transmission Electron Microscopy (STEM) mode. The results along Line 1 [Fig. 3(i)] suggest a diffusion profile for both copper, oxygen, and silver elements, indicating an inter-diffusion region as seen in Fig. 3(e). The results along Line 2 [Fig. 3(j)] were obtained at the top periphery region of the Ag nanowire. We could see an almost invariable copper and oxygen element distribution, suggesting that the Ag nanowire was embedded into the $\mathrm{CuO}$ nanowire region. It should be noted that as-synthesized $\mathrm{Ag}$ nanowires have a polyvinylpyrrolidone (PVP) ${ }^{22}$ protective coating to prevent agglomeration. However, this coating is likely to be removed by ablation in the initial stages of laser irradiation, and no residual organic material is found on the Ag nanowire at the $\mathrm{Ag}-\mathrm{CuO}$ nanowire interface after irradiation.

We further simulated the electric field distribution for the $\mathrm{Ag}-\mathrm{CuO}$ nanowire heterojunction when the laser irradiation polarization direction is along the long axis of the Ag nanowire, which agrees with the experimental results. In the simulation, the diameters of the $\mathrm{Ag}$ and $\mathrm{CuO}$ nanowire are $50 \mathrm{~nm}$ and $150 \mathrm{~nm}$, respectively, and each nanowire is $10 \mu \mathrm{m}$ in length. As seen in Figs. 4(a) and $4(\mathrm{~b})$, the simulation demonstrates that strong enhancement in the electric field occurred in the localized region at the $\mathrm{Ag}-\mathrm{CuO}$ nanowire heterojunction. We can also see slight enhancement of an electric field along the long axis of the Ag nanowire with a periodical distribution. This can lead to melting of $\mathrm{Ag}$ nanowires into some sphere structures when the laser irradiation fluence is too high or the irradiation time is too long, in agreement with the experimental results in Fig. 2(d). Since the fs laser pulse width is much shorter than the time for electron lattice thermal coupling and thermal diffusion to the lattice, excited electrons do not transfer energy to the lattice during the fs laser pulse. Under these conditions, which are possible only with ultrashort pulse irradiation, the input energy is initially localized in regions of high intensities as indicated by FEM simulations. Lattice heating is, therefore, initially concentrated in the regions of high optical field intensity. This results in strong spatially localized heating when the energy stored in the electron gas is transferred to the lattice, facilitating softening and melting of the lattice at these locations. ${ }^{24,27,28}$ Irradiation with fs laser pulses then permits selective heating of sub-wavelength volumes of the nano-component unlike irradiation with CW and long laser pulses that uniformly heat the entire volume of the component. In addition, multi-photon excitation is possible under excitation with high intensity fs laser radiation. We propose that fs laser heating of the $\mathrm{CuO}$ nanowire in the present (a)

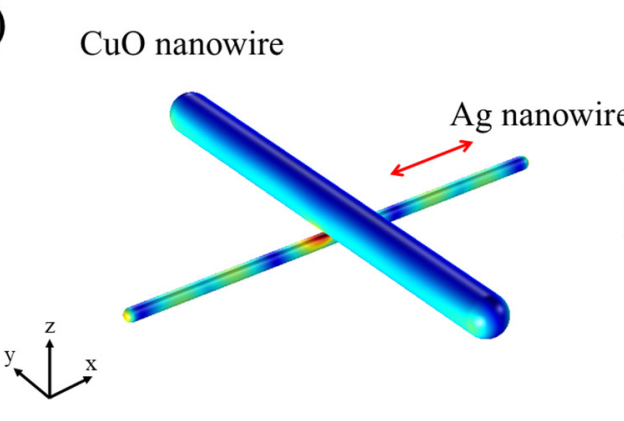

(c)

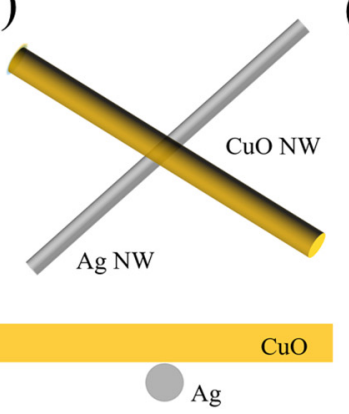

(d)

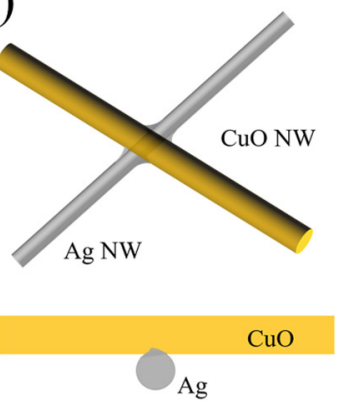

(b)
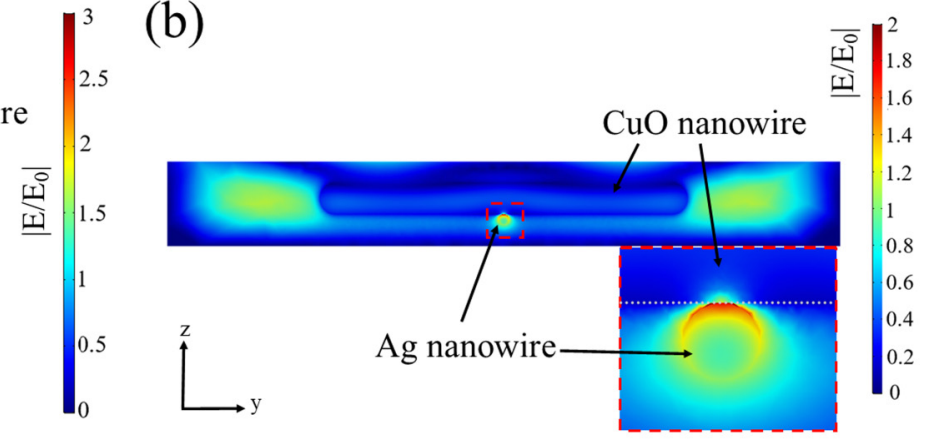

(e)

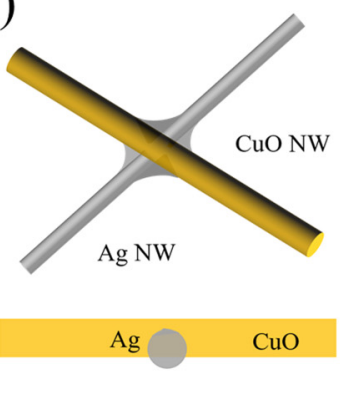

(f)

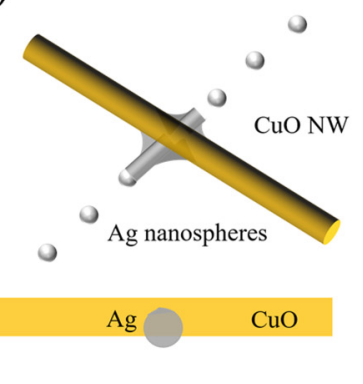

FIG. 4. FEM simulation showing the normalized electric field intensity distribution ( $\left|E / E_{0}\right|$, where $E_{0}$ is the incident field intensity) in and around a heterojunction between crossed $\mathrm{Ag}$ and $\mathrm{CuO}$ nanowires. (a) 3D view and (b) the view from the yz plane. The double arrow in (a) indicates the direction of laser polarization. The figure in the bottom right in (b) shows an expanded view at the junction. The dotted line in this figure shows the boundary between the CuO and Ag structures. (c)-(f) stages in the formation of the $\mathrm{Ag}-\mathrm{CuO}$ nanowire heterojunction under laser irradiation with increasing irradiation time (green arrow). The upper panels show a $3 \mathrm{D}$ view, while a schematic representation of joining in which the $\mathrm{Ag}$ nanowire becomes embedded in the $\mathrm{CuO}$ nanowire is shown in the lower panels. 
(a)

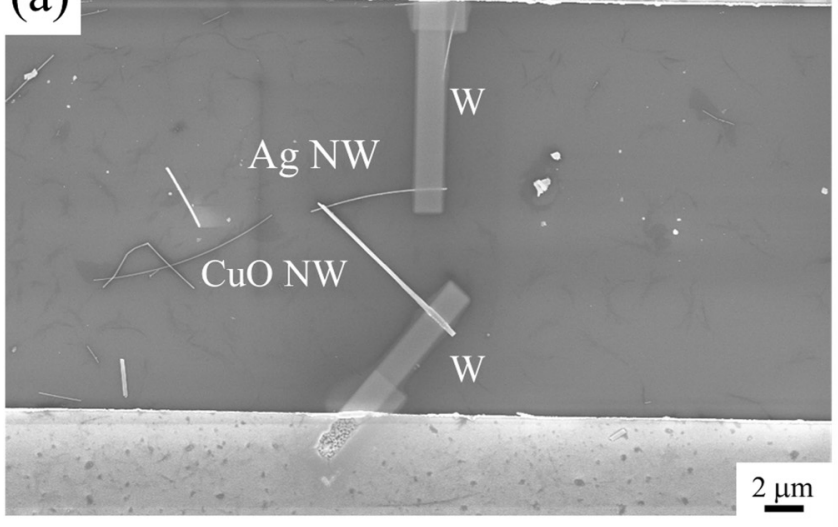

(c)

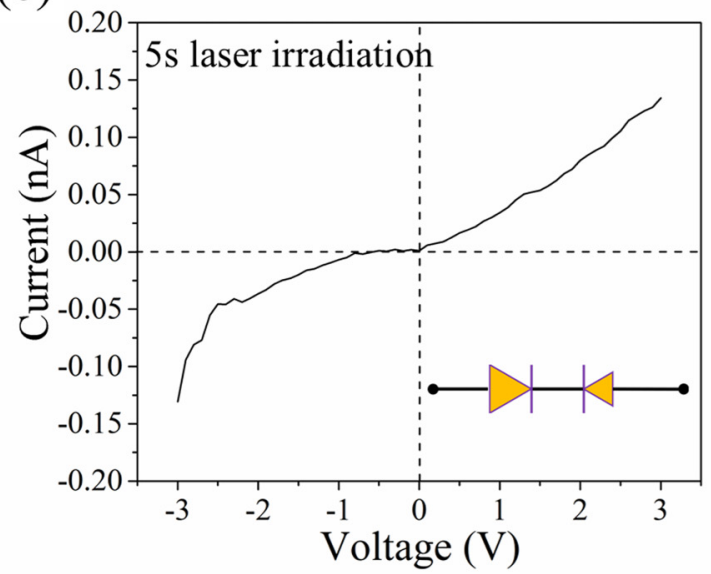

(e)

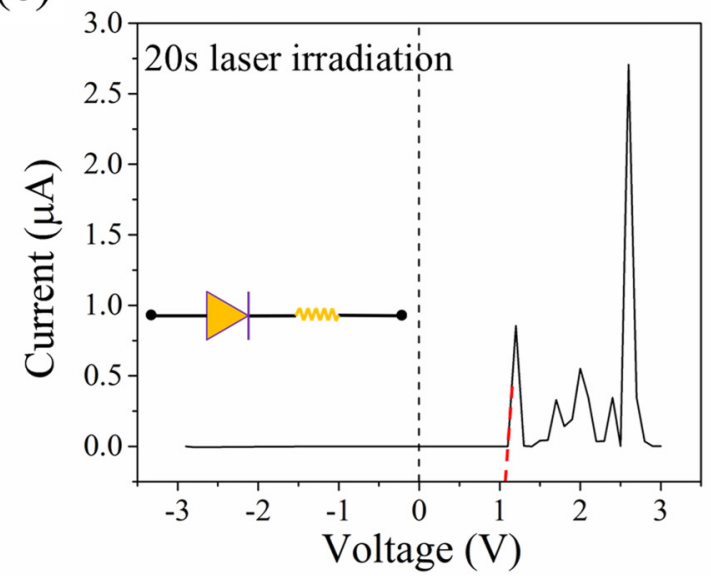

(b)

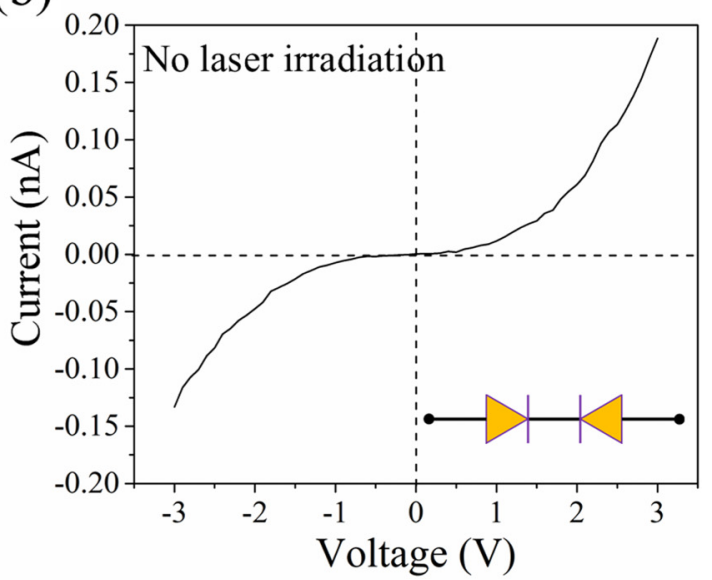

(d)

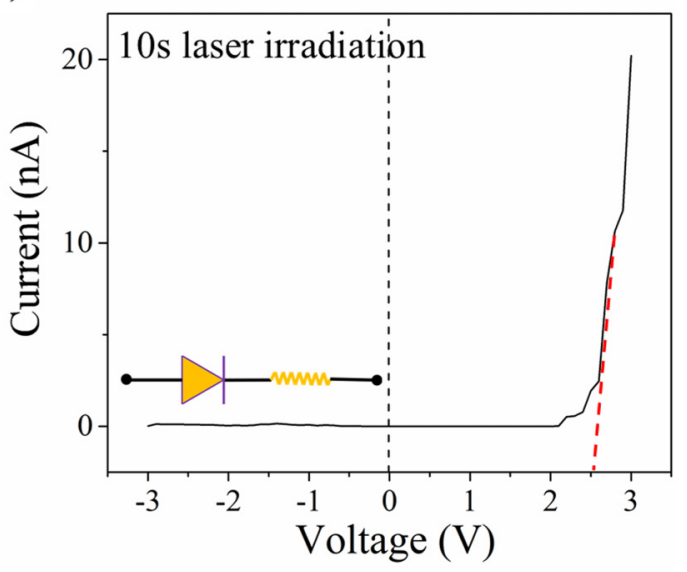

(f)

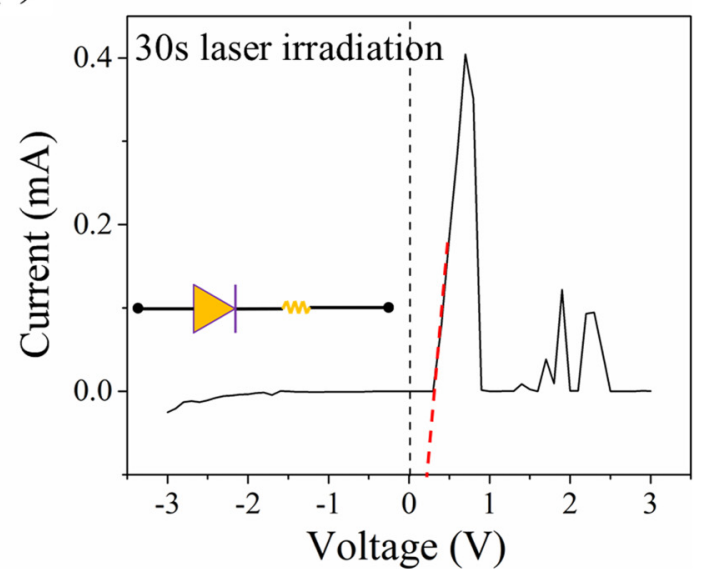

FIG. 5. (a) Ag-CuO nanowire heterojunction device fabricated by FIB induced W deposition on each end of the nanowire bridging to adjacent electrodes. Electrical characterization of the Ag-CuO nanowire heterojunction before (b) and after fs laser irradiation for (c) $5 \mathrm{~s}$, (d) $10 \mathrm{~s},(\mathrm{e}) 20 \mathrm{~s}$, and (f) $30 \mathrm{~s}$ at a fluence of $10 \mathrm{~mJ} / \mathrm{cm}^{2}$. The inset of the figure shows a schematic for the equivalent circuit under different stages of the fs laser irradiation. 
structures occurs in part via two-photon excitation of electrons from the valence band to conduction band. This creates a high density electron hole plasma that propagates toward the $\mathrm{Ag}-\mathrm{CuO}$ junction ${ }^{29,30}$ and selectively deposits energy at this location, resulting in softening of the $\mathrm{CuO}$ lattice, allowing the $\mathrm{Ag}$ nanowire to become embedded into the $\mathrm{CuO}$ nanowire. Overall, under fs laser irradiation, a combination of localized heating and melting of the $\mathrm{Ag}$ nanowire, together with softening of the $\mathrm{CuO}$ nanowire in the region of overlap, results in the observed bonding of the two nanowires and the formation of a junction localized at the interface between the two components. This process occurs without the generation of significant thermal heating in either nanowire outside the $\mathrm{Ag}-\mathrm{CuO}$ interface.

Based on the above discussion, a schematic diagram of the joining evolution process for the $\mathrm{Ag}-\mathrm{CuO}$ nanowire heterojunction under fs laser irradiation is shown in Figs. 4(c)-4(f). In the initial stage, the van Der Walls bonds exist for the $\mathrm{Ag}$ and $\mathrm{CuO}$ nanowire upon contact [Fig. 4(c)]. Fs laser irradiation leads to localized plasmonic electric field intensity at the $\mathrm{Ag}-\mathrm{CuO}$ nanowire interface, generating heat, which can promote the fuse of $\mathrm{Ag}$ and $\mathrm{CuO}$ nanowires [Fig. 4(d)]. Some of the Ag atoms begin to diffuse into the $\mathrm{CuO}$ nanowire at this stage. With longer laser irradiation time, the continuous $\mathrm{Ag}$ atom diffusion makes the $\mathrm{Ag}$ nanowire be partly embedded into the $\mathrm{CuO}$ nanowire. Furthermore, more Ag atoms will be fused and joined at the junction. Eventually, a high energy input due to extended laser irradiation causes the Ag nanowire to be broken down into $\mathrm{Ag}$ nanospheres, breaking down the $\mathrm{Ag}-\mathrm{CuO}$ nanowire heterojunction.

Tungsten (W) electrodes formed using FIB were used for fabrication of an $\mathrm{Ag}-\mathrm{CuO}$ nanowire heterojunction device [Fig. 5(a)]. Figure 5(b) shows the I-V characteristic of this junction prior to laser irradiation. It can be seen that the as-fabricated $\mathrm{W}-\mathrm{CuO}$ NW-Ag-W device exhibits an I-V response typical of a double-Schottky barrier. This can be associated with the presence of Schottky barriers at the $\mathrm{W} / \mathrm{CuO}$ and $\mathrm{Ag} / \mathrm{CuO}$ interfaces arising from the difference in the work function on each side of the interface. The PVP protection layer on the Ag nanowire surface might also present as a barrier for electron transport from the Ag electrode to the $\mathrm{CuO}$ nanowire. After laser irradiation for $5 \mathrm{~s}$, there is little change in the reverse current compared to that of the unirradiated device. However, after irradiation, the forward current develops a quasi-linear response consistent with a reduction in the Schottky barrier at the $\mathrm{Ag} / \mathrm{CuO}$ interface [Fig. 5(c)]. This decrease could arise as $\mathrm{Ag}$ atoms are introduced into the $\mathrm{CuO}$ structure in response to fs laser irradiation, as Ag doping will lower the bandgap energy in $\mathrm{CuO}$. A theoretical calculation via density functional theory indicates that increasing the Ag dopant concentration to $3.125 \%$ lowers the bandgap energy in $\mathrm{CuO}$ from $1.99 \mathrm{eV}$ to $1.152 \mathrm{eV}^{31}$ The diffusion of $\mathrm{Ag}$ atoms in $\mathrm{CuO}$ will also lead to an increase in the probability of inter-band electronic excitation, which will promote electron transfer from the Ag nanowire to the $\mathrm{CuO}$ nanowire, effectively lowering the Schottky barrier at the $\mathrm{Ag} / \mathrm{CuO}$ nanowire interface. As the fs laser irradiation time increases to $10 \mathrm{~s}$, the device is converted into a rectifier and the forward current dramatically increases [Fig. 5(d)]. This is consistent with a reduction of the Schottky barrier and the creation of an Ohmic contact. As additional $\mathrm{Ag}$ atoms diffuse into the bulk of the $\mathrm{CuO}$ nanowire with further fs laser irradiation, an interdiffusion layer appears as shown in the HRTEM image in Fig. 3(e). This produces a highly doped $\mathrm{CuO}$ layer at the $\mathrm{Ag}-\mathrm{CuO}$ nanowire interface. A heterojunction is formed when the depletion layer at the $\mathrm{Ag} / \mathrm{CuO}$ interface is sufficiently narrow, assisting direct carrier tunneling at the interface $^{32}$ and forming an ohmic contact. ${ }^{33}$ To characterize this behavior, we have used a conventional piecewise linear model to analyze the forward current behavior in our devices. This model shows that the turn-on voltage decreases from $\sim 2.5 \mathrm{~V}$ to $\sim 0.3 \mathrm{~V}$ as the fs laser irradiation time increases from $10 \mathrm{~s}$ to $30 \mathrm{~s}$. The resistance, $\mathrm{R}$, obtained from the slope of the line segment tangent to the diode curve, is $\sim 2.7 \times 10^{7}, \sim 1.2 \times 10^{5}$, and $\sim 1.0 \times 10^{3} \Omega$ in Figs. 5(d)-5(f), respectively. The large reduction in the effective resistance of the diode with increasing laser irradiation time likely arises as $\mathrm{Ag}$ atoms diffuse into the $\mathrm{CuO}$ nanowire and act as a dopant, increasing the conductivity in the $\mathrm{Ag} / \mathrm{CuO}$ nanowire interface. The current spikes observed when $\mathrm{V}$ exceeds the threshold voltage in heavily irradiated devices [Figs. 5(e) and 5(f)] can be associated with the migration of $\mathrm{Ag}$ toward the $\mathrm{Ag} / \mathrm{CuO}$ interface induced by the high electric field. An increase in the laser irradiation time to $40 \mathrm{~s}$ leads to mechanical instability and dissolution of the heterojunction as the $\mathrm{Ag}$ nanowire breaks into a series of nanospheres. A similar response was found in all samples.

\section{CONCLUSIONS}

We show that the interface and electrical characteristics of an $\mathrm{Ag}-\mathrm{CuO}$ nanowire heterojunction can be readily engineered by processing with fs laser radiation. Exposure to a short period of low fluence fs laser pulses results in a structure in which the Ag nanowire remains intact but becomes embedded into the $\mathrm{CuO}$ nanowire. This effect occurs as a combination of plasmonic heating in the Ag nanowire and excitonic heating in the $\mathrm{CuO}$ nanowire induces surface melting in the $\mathrm{Ag}$ nanowire and softening of the $\mathrm{CuO}$ nanowire. Meanwhile, the $\mathrm{Ag} / \mathrm{CuO}$ interface reconstructs to yield lattice matching based on $\mathrm{Ag}(111) \| \mathrm{CuO}(111)$, while both the $\mathrm{Ag}$ and $\mathrm{CuO}$ nanowires retain their single crystalline structures away from the interfacial region. Devices fabricated as a $\mathrm{W}-\mathrm{CuO}-\mathrm{Ag}-\mathrm{W}$ heterojunction undergo a transition from double-Schottky contact behavior to a typical rectifying behavior after fs laser processing. Furthermore, the interdiffusion of atoms at the $\mathrm{Ag} / \mathrm{CuO}$ interface promotes charge transfer between the metal and semiconductor, which reduces the resistance of this heterojunction, further increasing the forward current for the rectifying behavior. Overexposure to laser radiation produces damage in the $\mathrm{Ag}$ nanowire, which results in breakage and dissolution into Ag nanoparticles, breaking down the $\mathrm{Ag}-\mathrm{CuO}$ nanowire heterojunction. This study demonstrates that fs laser processing can be used for precise engineering of a nanoscale interface and tailoring of the electrical performance of a nanoscale device incorporating this junction.

\section{ACKNOWLEDGMENTS}

This work was supported by the Natural Sciences and Engineering Research Council (NSERC) and the Canada Research Chairs (CRC) Programs. 


\section{DATA AVAILABILITY}

The data that support the findings of this study are available from the corresponding author upon reasonable request.

\section{REFERENCES}

${ }^{1}$ C. Cao, J. B. Andrews, A. Kumar, and A. D. Franklin, ACS Nano 10, 5221 (2016).

${ }^{2}$ P. N. Nirmalraj and J. J. Boland, ACS Nano 4, 3801 (2010).

${ }^{3}$ Y. F. Lin and W. B. Jian, Nano Lett. 8, 3146 (2008).

${ }^{4}$ A. M. Lord, T. G. Maffeis, O. Kryvchenkova, R. J. Cobley, K. Kalna, D. M. Kepaptsoglou, Q. M. Ramasse, A. S. Walton, M. B. Ward, J. Köble, and S. P. Wilks, Nano Lett. 15, 4248 (2015).

${ }^{5}$ A. M. Lord, Q. M. Ramasse, D. M. Kepaptsoglou, J. E. Evans, P. R. Davies, M. B. Ward, and S. P. Wilks, Nano Lett. 17, 687 (2017).

${ }^{6}$ D. Lynall, S. V. Nair, D. Gutstein, A. Shik, I. G. Savelyev, M. Blumin, and H. E. Ruda, Nano Lett. 18, 1387 (2018).

${ }^{7}$ Y. Guo, Y. Han, J. Li, A. Xiang, X. Wei, S. Gao, and Q. Chen, ACS Nano 8, 7771 (2014).

${ }^{8}$ Z. Yang, C. Kim, K. Y. Lee, M. Lee, S. Appalakondaiah, C. H. Ra, K. Watanabe, T. Taniguchi, K. Cho, E. Hwang, J. Hone, and W. J. Yoo, Adv. Mater. 31, 1808231 (2019).

${ }^{\mathbf{9}}$ F. Xia, V. Perebeinos, Y. M. Lin, Y. Wu, and P. Avouris, Nat. Nanotechnol. 6, 179 (2011).

${ }^{10^{Y}}$. Liu, J. Guo, E. Zhu, L. Liao, S. J. Lee, M. Ding, I. Shakir, V. Gambin, Y. Huang, and X. Duan, Nature 557, 696 (2018).

${ }^{11}$ J. Zhang, K. Zhang, B. Xia, Y. Wei, D. Li, K. Zhang, Z. Zhang, Y. Wu, P. Liu, X. Duan, Y. Xu, W. Duan, S. Fan, and K. Jiang, Adv. Mater. 29, 1702942 (2017).

${ }^{12}$ P. Ghosh, J. Lu, Z. Chen, H. Yang, M. Qiu, and Q. Li, Adv. Electron. Mater. 4, 1700614 (2018)

${ }^{13}$ S. Xing, L. Lin, G. Zou, L. Liu, P. Peng, A. Wu, W. W. Duley, and Y. N. Zhou, Nanotechnology 28, 405302 (2017).

${ }^{14}$ L. Lin, L. Liu, K. Musselman, G. Zou, W. W. Duley, and Y. N. Zhou, Adv. Funct. Mater. 26, 5979 (2016).
${ }^{15}$ S. Xing, L. Lin, J. Huo, G. Zou, X. Sheng, L. Liu, and Y. N. Zhou, ACS Appl. Mater. Interfaces 11, 9326 (2019).

${ }^{16}$ J. Ha, B. J. Lee, D. J. Hwang, and D. Kim, RSC Adv. 6, 86232 (2016).

${ }^{17}$ S. Ding, Y. Tian, Z. Jiang, and C. Wang, Mater. Trans. 56, 981 (2015).

${ }^{18}$ Y. Hu, C. Liang, X. Sun, J. Zheng, J. Duan, and X. Zhuang, Nanomaterials 9 , 673 (2019).

${ }^{19} \mathrm{G}$. González-Rubio, J. González-Izquierdo, L. Bañares, G. Tardajos, A. Rivera, T. Altantzis, S. Bals, O. Peña-Rodríguez, A. Guerrero-Martínez, and L. M. Liz-Marzán, Nano Lett. 15, 8282 (2015).

${ }^{20} \mathrm{H}$. Yu, X. Li, Z. Hao, W. Xiong, L. Guo, Y. Lu, R. Yi, J. Li, X. Yang, and X. Zeng, Nanoscale 9, 7012 (2017).

${ }^{21}$ X. Jiang, T. Herricks, and Y. Xia, Nano Lett. 2, 1333 (2002).

${ }^{22}$ Z. Hui, Y. Liu, W. Guo, L. Li, N. Mu, C. Jin, Y. Zhu, and P. Peng, Nanotechnology 28, 285703 (2017).

${ }^{23}$ L. Lin, G. Zou, L. Liu, W. W. Duley, and Y. N. Zhou, Appl. Phys. Lett. 108, 203107 (2016).

${ }^{24}$ L. Liu, P. Peng, A. Hu, G. Zou, W. W. Duley, and Y. N. Zhou, Appl. Phys. Lett. 102, 073107 (2013).

${ }^{25}$ P. Ghosh, J. Lu, H. Luo, Z. Xu, X. Yan, Y. Wang, J. Lu, M. Qiu, and Q. Li, Opt. Lett. 43, 2422 (2018).

${ }^{\mathbf{2 6}} \mathrm{H}$. Oh, J. Lee, and M. Lee, Appl. Surf. Sci. 427, 65 (2018).

${ }^{27}$ A. Hu, G. L. Deng, S. Courvoisier, O. Reshef, C. C. Evans, E. Mazur, and Y. Zhou, Proc. SPIE 8809, 880907 (2013).

${ }^{28}$ A. Hu, P. Peng, H. Alarifi, X. Y. Zhang, J. Y. Guo, Y. N. Zhou, and W. W. Duley, J. Laser Appl. 24, 042001 (2012).

${ }^{29} \mathrm{D}$. von der Linde, K. Sokolowski-Tinten, and J. Bialkowski, Appl. Surf. Sci. 109-110, 1 (1997).

${ }^{30}$ Z. Jiao, H. Huang, L. Liu, A. Hu, W. Duley, P. He, and Y. Zhou, J. Appl. Phys. 115, 134305 (2014).

${ }^{31}$ H. Absike, M. Hajji, H. Labrim, A. Abbassi, and H. Ez-Zahraouy, Superlattices Microstruct. 127, 128 (2019).

${ }^{32}$ S. M. Sze and K. K. Ng, Physics of Semiconductor Devices (John Wiley \& Sons, 2006).

${ }^{33}$ G. A. Hope and A. J. Bard, J. Phys. Chem. 87, 1979 (1983). 